\title{
An Effective Silencer Design for Artificially Air Conditioned Environment
}

\author{
Kyoji Fujiwara $^{1)}$ and Li Feng Pang ${ }^{2)}$ \\ 1) Department of Acoustics, Faculty of Design, Kyushu University School of Design \\ 2) School of Design, Kyushu Institute of Design
}

\begin{abstract}
An effective silencer for an air conditioning duct is studied. A duct with an acoustically soft boundary is employed as an effective silencer. On the acoustically soft boundary the sound pressure is zero and it is impossible to realize such boundary in the air-borne sound field, because of the nonexistence of a much lighter medium than the air. In this study, the arrangement of one-quarter wave-length acoustic tubes is employed as a soft boundary. This acoustic tube has frequency dependence, but the sound pressure becomes nearly zero at the tube mouth around the odd resonance frequency. The relation between the noise reduction efficiency and this acoustically soft boundary is examined experimentally and more than $40 \mathrm{~dB}$ noise reduction is obtained in a one-half octave band around the first resonance frequency. It is also made clear that more than one wave length of soft boundary is required to get enough reduction compared with the reduction obtained in the case of quite a long soft boundary. J Physiol Anthropol Appl Human Sci 23(6): 231-235, 2004 http://www.jstage.jst.go.jp/ browse/jpa
\end{abstract}

Keywords: duct, acoustic soft boundary, noise reduction

\section{Introduction}

In buildings, there are many noise sources. Air conditioning machines especially produce high level noise and an air duct supplying fresh cool or warm air also carries noise. In the low frequency range, the noise can propagate quite easily through the duct. Therefore in rooms required to meet high level environmental specifications, noise control also plays an important role. So far, the machine noise could be reduced by an absorbing duct or an absorbing elbow. For these silencers fibrous material is used. This fibrous material can fly into pieces and be carried into the room. Also this material can absorb sound in the high frequency range, but not so much in low frequency range. Therefore an effective noise silencer in the low frequency range is required.

In this study, an acoustically soft boundary is employed to construct the duct. Usually the duct is constructed of a hard plate like a thin steel plate and fibrous material fixed to the wall inside is used for noise reduction. It is already known that low frequency sound can not propagate in the duct, when the duct wall boundary is acoustically soft. Here, the arrangement of one-quarter wave length acoustic tubes is used as a soft acoustic boundary. At the mouth of the acoustic tube the sound pressure becomes nearly zero around the odd resonance frequencies of the tube. So far one-quarter wave length acoustic tubes have been used as a side branch to reduce duct noise, but only one tube has been used (Radavich and Selamet, 2001). The arrangement of many tubes has not been used as a soft boundary. Here, the relation between the noise reduction efficiency and the acoustically soft boundary is examined under the conditions of no wind in the duct.

\section{Theory}

Generally the air duct with an acoustically soft boundary has not been studied because it is very hard to realize the soft boundary. Some sound fields have been analyzed theoretically, but in these cases, the sound field in the duct with a soft boundary is trivial because the sound can not propagate through the duct (Skudrzyk, 1971). Here, this fact is positively employed and to realize the soft boundary is examined. The air duct is usually constructed of thin steel plates. At the steel plate surface the sound pressure is finite and the particle velocity normal to the surface is zero. But at the acoustically soft surface the sound pressure is zero and the particle velocity is finite. The sound fields in these ducts are briefly introduced.

Let the duct be rectangular and the size of cross-section be $a \times b$ as shown in Fig. 1. The $z$ axis is the direction of duct air flow. A solution of the sound wave equation expressed in Cartesian coordinates for a plane sine wave incidence is shown by Eq. (1). The time dependence $\exp (j \omega t)$ is omitted.

$$
\begin{aligned}
& p(x, y, z)= A\left\{\exp \left(j k_{x} x\right)+R_{x} \exp \left(-j k_{x} x\right)\right\}\left\{\exp \left(j k_{y} y\right)+R_{y} \exp \left(-j k_{y} y\right)\right\} \\
& \times\left\{\exp \left(j k_{z} z\right)+R_{z} \exp \left(-j k_{z} z\right)\right\} \\
& k_{x}^{2}+k_{y}^{2}+k_{z}^{2}=k^{2}
\end{aligned}
$$

Here, $A, R_{x}, R_{y}, R_{z}$ are the amplitude and the reflection coefficients in the $x, y, z$ direction respectively. $k$ is the wave 


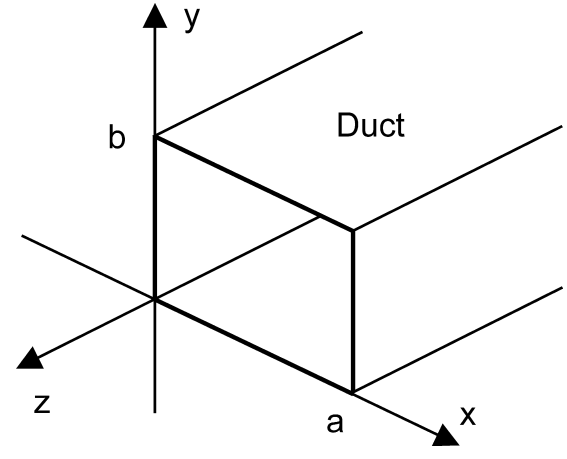

Fig. 1 Rectangular duct size and coordinates

number and $j$ is the imaginary unit. At the first, let four boundaries around the duct be acoustically hard, that is, the normal particle velocities at surfaces are zero. The boundary conditions are expressed as follows.

$$
\begin{array}{ll}
v_{x}=0: y=0, b, & 0<x<a \\
v_{y}=0: x=0, a, & 0<x<b
\end{array}
$$

The solution for this boundary value problem is shown by Eq. (2).

$$
\begin{gathered}
p(x, y, z)=\sum_{m} \sum_{n} A_{m n} \cos \left(\frac{m \pi}{a} x\right) \cos \left(\frac{n \pi}{b} y\right) \exp \left(-j k_{z m n} z\right) \\
k_{z m n}^{2}=k^{2}-\left(\frac{m \pi}{a}\right)^{2}-\left(\frac{n \pi}{b}\right)^{2}
\end{gathered}
$$

In the case of the low frequency problem, the size of duct is usually much smaller than the wave-length. In this case $m$ and $n$ in Eq. (2) becomes zero and the amplitude of the wave in the $z$ direction becomes $A_{00}$ and a plane wave can propagate. When a pair of duct surfaces is acoustically soft, for example a pair of surfaces parallel to the $y$ axis, then the boundary conditions are as follows;

$$
\begin{array}{ll}
v_{x}=0: y=0, b, & 0<x<a \\
p=0: x=0, a, & 0<y<b
\end{array}
$$

In this case the solution is expressed by Eq. (3).

$$
p(x, y, z)=\sum_{m} \sum_{n} A_{m n} \sin \left(\frac{m \pi}{a} x\right) \cos \left(\frac{n \pi}{b} y\right) \exp \left(-j k_{z m n} z\right)
$$

Also in the case of low frequency problems, $m$ and $n$ become zero and the amplitude of the wave in the $z$ direction becomes zero because of the existence of the sin function. In this case no plane wave propagates in the $z$ direction. To take another case, a pair of duct surfaces parallel to the $x$ axis is acoustically soft and the other pair is acoustically hard. The

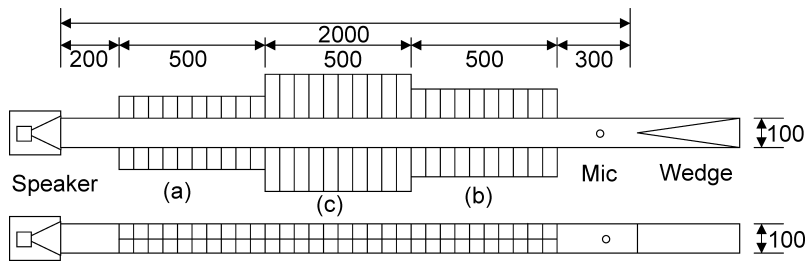

Fig. 2 An arrangement of one-quarter wave-length acoustic tubes as an acoustically soft boundary.

acoustical condition is similar to the case shown just above and no low frequency plane wave can propagate. Finally, when both pair of duct surfaces are acoustically soft, the boundary conditions are given by,

$$
\begin{array}{ll}
p=0: y=0, b, & 0<x<a \\
p=0: x=0, a, & 0<y<b
\end{array}
$$

and the solution is given by Eq. (4).

$$
p(x, y, z)=\sum_{m} \sum_{n} A_{m n} \sin \left(\frac{m \pi}{a} x\right) \sin \left(\frac{n \pi}{b} y\right) \exp \left(-j k_{z m n} z\right)
$$

In this case, the amplitude of the wave becomes much smaller than the amplitudes in the case mentioned above because of the existence of double sin functions. Of course no plane wave can propagate in the $z$ direction. As shown in these cases, if a pair of duct surfaces is acoustically soft, then no low frequency plane wave propagates through the duct.

\section{Realization of the soft boundary and measurement of the effect}

At the acoustically soft surface the sound pressure is zero for all over the frequency range. It is the only way to satisfy this condition to consist the boundary with a medium very much lighter than the air. It is really impossible. Another way is frequency dependent, but it realizes the soft boundary for a certain frequency range. It is to arrange many one-quarter wave length acoustic tubes at the boundary. An example of this arrangement is shown in Fig. 2. Twenty tubes of $50 \mathrm{~mm} \times$ $50 \mathrm{~mm}$ cross-section are arranged to construct the $100 \mathrm{~mm} \times$ $500 \mathrm{~mm}$ acoustically soft boundary surface. The depth of each tube is $130 \mathrm{~mm}$ which is one-quarter wave length of a $650 \mathrm{~Hz}$ sound wave. These kinds of units are also constructed for different design frequencies, that is, $500 \mathrm{~Hz}$ and $1 \mathrm{kHz}$. These units are named (a) for $1 \mathrm{kHz}$, (b) for $650 \mathrm{~Hz}$ and (c) for $500 \mathrm{~Hz}$. An acoustically hard boundary is also constructed of a $20 \mathrm{~mm}$ thickness acrylic plate and named (o).

As shown in Fig. 3, these different acoustic boundaries were set along the test duct of $100 \mathrm{~mm} \times 100 \mathrm{~mm}$ cross-section and the effect of these boundaries on noise reduction was measured. The length of this duct is $2000 \mathrm{~mm}$ and it can accept 3 sets of acoustic boundary unit arrangements. The end of the 


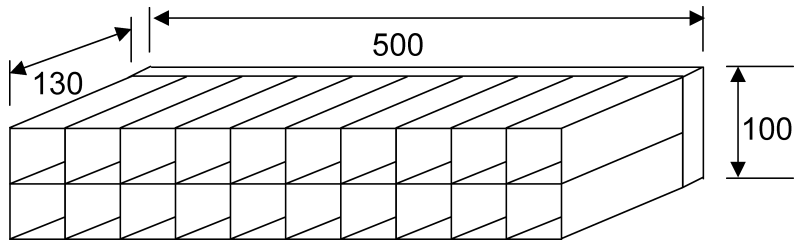

Fig. 3 Test facility for the measurement of noise reduction efficiency obtained by the soft duct constructed by the tube arrangements.

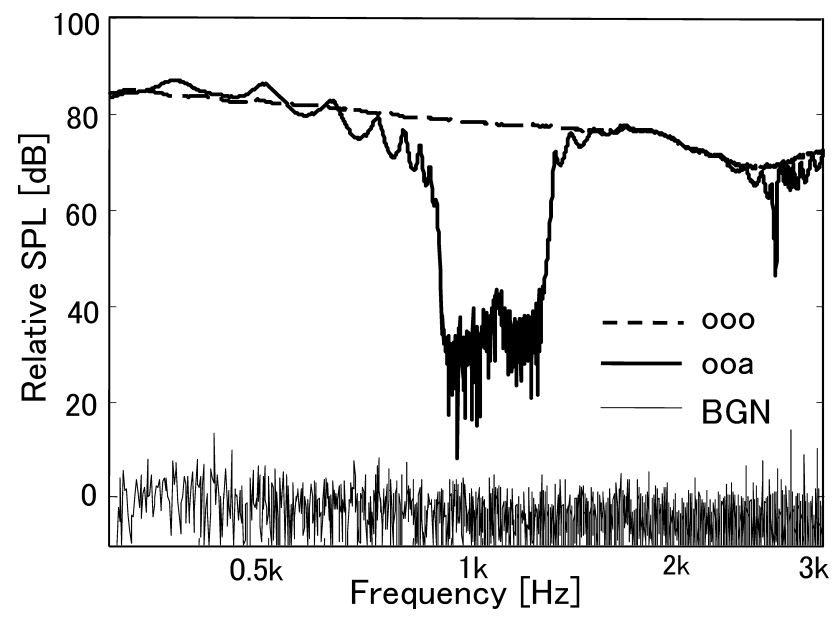

Fig. 4 An example of the measured sound reduction efficiency.

receiver side was terminated by an absorbing wedge of $99.9 \%$ absorption and just in front of the wedge a microphone was set. A loud speaker was set at the other terminal and a noise signal was produced. Figure 4 shows an example of the measured result when a pair of soft surface units (a), named (ooa), was set. The dashed curve shows the relative sound pressure level at the receiving point, that is, in the case where the whole boundary was hard, named case (ooo). The thick solid curve shows a sound pressure level when a pair of units (a) was installed, that is, the length of the soft boundary is only $50 \mathrm{~cm}$. We can see very high noise reduction efficiency around a design frequency of $1 \mathrm{kHz}$. It amounts to more than $40 \mathrm{~dB}$. The thin solid complex curve shows background noise level and it indicates that the measurement was done accurately.

\section{Results of measurements}

Figures 5-A, -B and $-\mathrm{C}$ show the measured results in the cases of soft boundary arrangement (a), (b) and (c), respectively. In Fig. 5-A, the thin solid curve shows the noise reduction efficiency in the case of (ooa), that is, only one pair of $50 \mathrm{~cm}$ soft boundary units was set. The ordinate shows the relative sound pressure level to the sound pressure level in the case of the hard boundary. The thick solid curve shows the reduction in the case of the arrangement (oaa), that is, $1 \mathrm{~m}$ soft and $50 \mathrm{~cm}$ hard. There is no big difference between both cases and $0.5 \mathrm{~m}$ soft boundary length is sufficient to effect a large reduction for a $1 \mathrm{kHz}$ sound wave. In Fig. 5-B, two solid curves show the reduction efficiency in the cases of (oob) and (obb). The noise
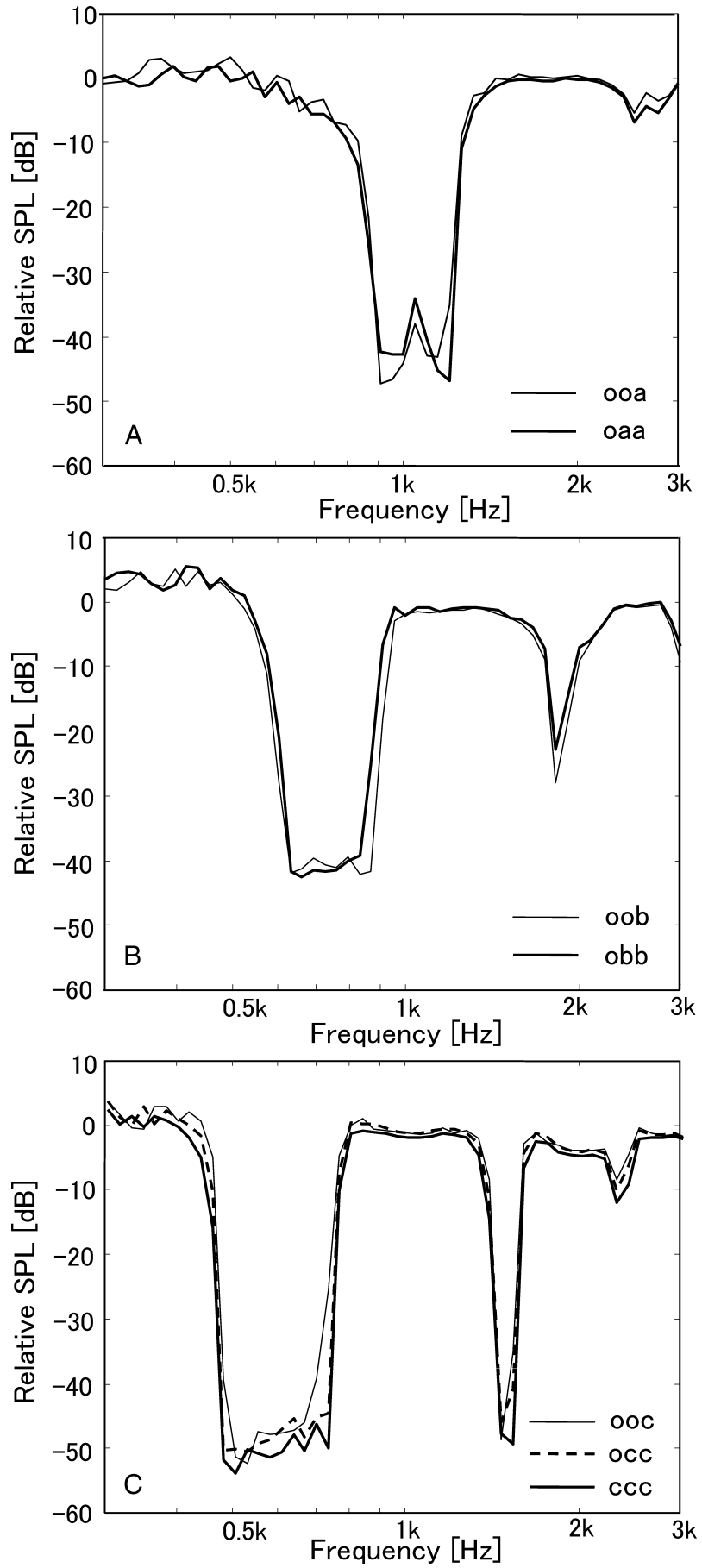

Fig. 5 Measured results of sound reduction efficiency. Figures 5-A, -B and $-\mathrm{C}$ show the results in the cases of soft boundary units (a), (b) and (c), respectively.

reduction efficiency is quite large around $650 \mathrm{~Hz}$ and it amounts also $40 \mathrm{~dB}$. Around $1950 \mathrm{~Hz}$, there is also a large reduction and it is caused by the next higher odd resonance of the acoustic tube. But there is also no big difference between both curves. In Fig. 5-C, the thin solid curve, the dashed curve and thick solid curve show the reduction efficiency in the cases of (ooc), (occ) and (ccc), respectively. In these cases, the 

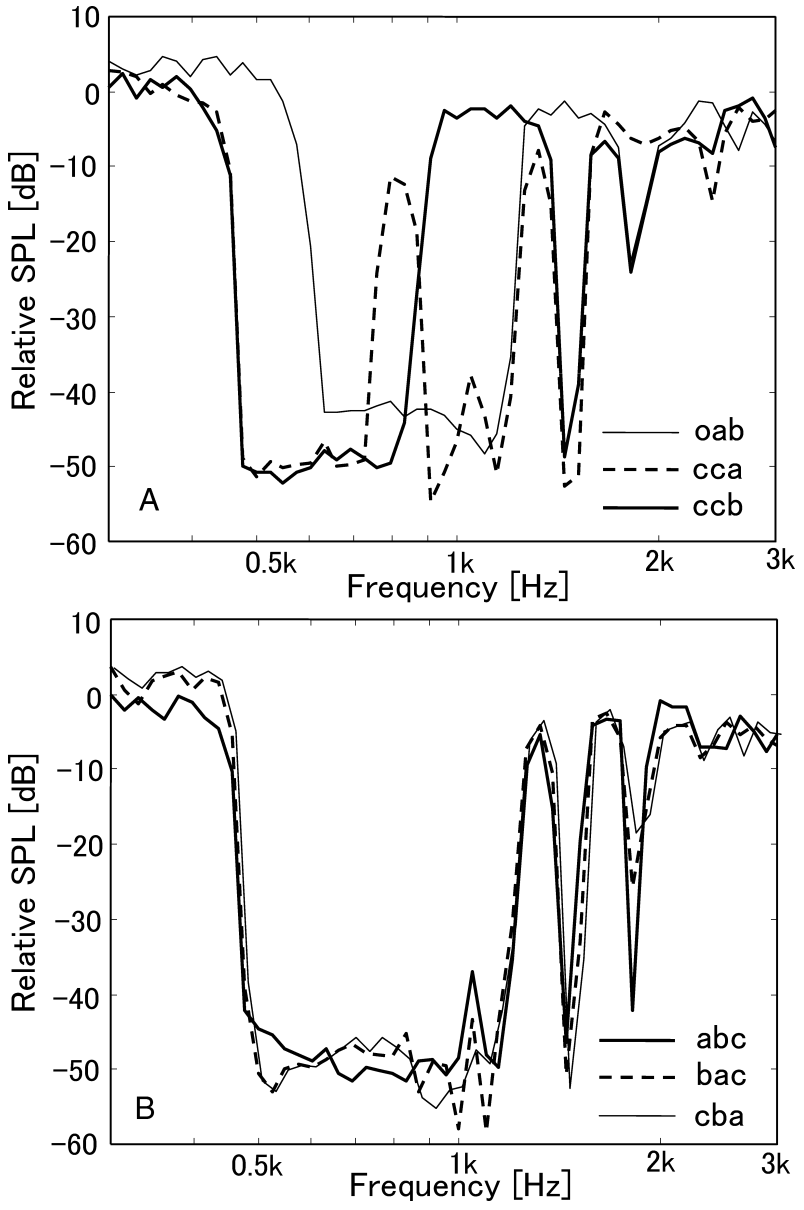

Fig. 6 Measured results of sound reduction efficiency. Figures 6-A and B show the results in the cases where two and three different kinds of units were connected in series, respectively.

reduction amounts to $50 \mathrm{~dB}$ around the design frequency. Some small difference could be found between the cases of (ooc) and (ccc). It might mean that a $50 \mathrm{~cm}$ soft boundary is not enough to reduce a $500 \mathrm{~Hz}$ sound wave effectively.

Next, the additivity in each efficiency obtained here was checked. The results are shown in Figs. 6-A and -B. In Fig. 6A, the thin solid, dashed and thick solid curves show the reduction efficiency in the case of (oab), (cca) and (ccb). In each case, the resultant reduction efficiency of cases (oab), (cca) and (ccb) are almost equal to the sum of reduction efficiency in the cases of (ooa) and (oob), (ooa) and (occ), (oob) and (occ) respectively. In Fig. 6-B, the curves of noise reduction efficiency in the cases of (abc), (bac) and (cba) show almost the same frequency characteristics and are equal to the sum of reduction in the cases of (ooa), (oob) and (ooc). The arrangement order of soft surface designed in different frequencies has no effect on the reduction efficiency. In these cases, the maximum reduction amounts to $50 \mathrm{~dB}$. From these data, the additivity of the reduction efficiency is supported.

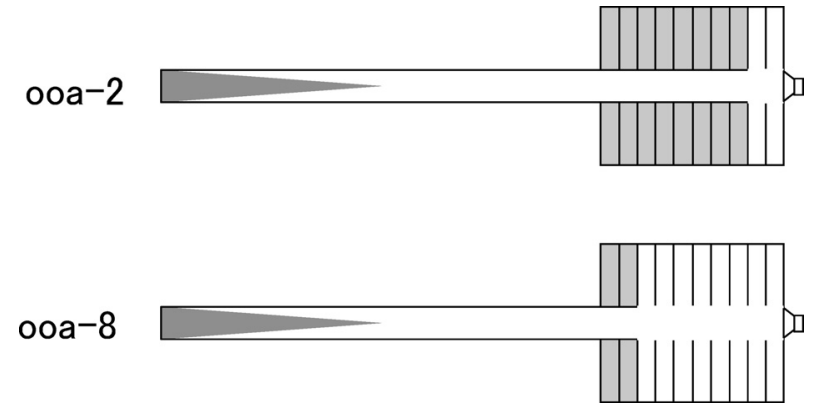

Fig. 7 Examples of different conditions of two and eight tube mouths being closed.

\section{Required length for sufficient reduction efficiency}

In the previous section, the relation between the soft surface and the reduction efficiency was studied. Here, the relation between the length of the soft surface boundary and the reduction efficiency is studied. As shown in Fig. 7, some openings of the soft boundary unit (a) were covered by square plates and the reduction efficiency was measured. For example, (ooa-4) means that 4 openings out of 10 are not covered and the other 6 openings are covered by plates. The measured results are shown in Figs. 8-A and -B. In Fig. 8-A, the dotted, thick solid, thick dashed and thin solid curves show the reduction efficiency in the case of (ooa-2), (ooa-4), (ooa-6) and (ooa) respectively. In Fig. 8-B, the thick solid, thick dashed and thin solid curves show the results in the cases of (ooa-7), (ooa8 ) and (ooa). From these data, when four openings are covered, that is, in the case of (ooa-6), the reduction efficiency would begin to decrease. When 3 openings are covered, the reduction efficiency is not much different from the case of (ooa), i.e., all 10 openings are not covered. These 7 openings are about $35 \mathrm{~cm}$ in length and this is nearly equal to one wave-length of $1 \mathrm{kHz}$ sound wave $(34 \mathrm{~cm})$. Thus it could be said that the soft boundary length of more than one wave-length is required to get sufficient reduction efficiency compared with the efficiency obtained in the case of quite a long soft surface boundary. This result has been also verified by the numerical examinations.

\section{Conclusions}

Here, the noise reduction efficiency of the duct constructed by the acoustically soft boundary was experimentally examined and the following conclusions were obtained.

(1) It is possible to construct an acoustically soft boundary by the arrangement of one-quarter wave-length acoustic tubes, even though it has frequency dependence.

(2) When one pair of parallel walls of a square duct is acoustically soft, more than $40 \mathrm{~dB}$ noise reduction is obtained in the frequency range about one-half octave band around the design frequency.

(3) The noise reduction efficiencies designed in the different frequencies could be added and a large noise reduction efficiency could be realized over a wide frequency range. 

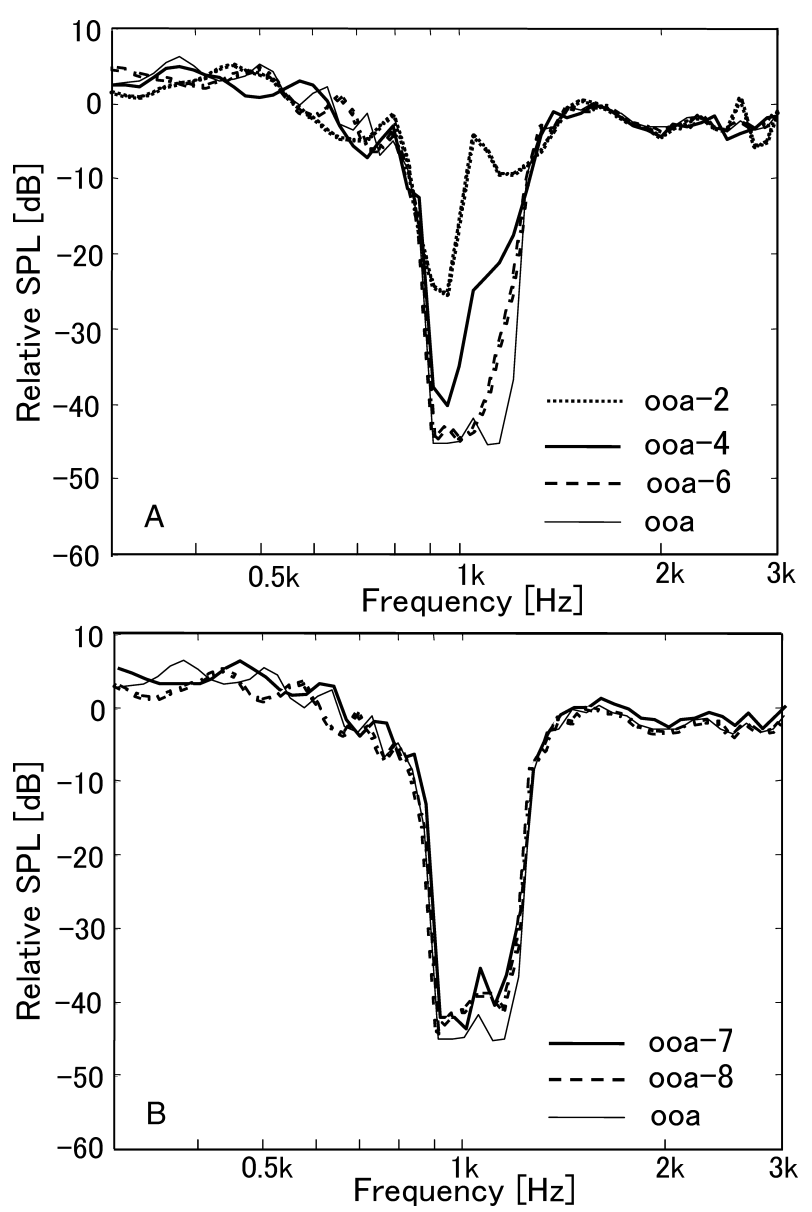

Fig. 8 Measured results of sound reduction efficiency for different lengths of soft boundary. Figure 8-A shows the results in the cases of soft boundary length 10, 20,30,50 cm, and Fig. 8-B in the case of 35, $40,50 \mathrm{~cm}$.
(4) More than one-wave length of soft boundary is required to get enough reduction compared with the reduction obtained in the case of quite a long soft boundary.

\section{References}

Radavich PM, Selamet A (2001) A computational approach for flow acoustic coupling in closed side branches. J Acoust Soc Am 109: 1343-1353

Skudrzyk E (1971) Foundation of Acoustics. Springer-Verlag, $431-432$

Received: September 3, 2004

Accepted: September 17, 2004

Correspondence to: Kyoji Fujiwara, Department of Acoustics, Faculty of Design, Kyushu Universiy School of Design, Shiobaru 4-9-1, Minami, Fukuoka 815-8540, Japan

Phone: $+81-92-553-4552$

Fax: $+81-92-553-4552$

e-mail: fujiwara@design.kyushu-u.ac.jp 